Review

\title{
From Hahn-Banach Type Theorems to the Markov Moment Problem, Sandwich Theorems and Further Applications
}

\section{Octav Olteanu}

Department of Mathematics-Informatics, University Politehnica of Bucharest, 060042 Bucharest, Romania; octav.olteanu50@gmail.com or olteanuoctav@yahoo.ie

Received: 23 July 2020; Accepted: 5 August 2020; Published: 10 August 2020

\begin{abstract}
The aim of this review paper is to recall known solutions for two Markov moment problems, which can be formulated as Hahn-Banach extension theorems, in order to emphasize their relationship with the following problems: (1) pointing out a previously published sandwich theorem of the type $f \leq h \leq g$, where $f,-g$ are convex functionals and $h$ is an affine functional, over a finite-simplicial set $X$, and proving a topological version for this result; (2) characterizing isotonicity of convex operators over arbitrary convex cones; giving a sharp direct proof for one of the generalizations of Hahn-Banach theorem applied to the isotonicity; (3) extending inequalities assumed to be valid on a small subset, to the entire positive cone of the domain space, via Krein-Milman or Carathéodory's theorem. Thus, we point out some earlier, as well as new applications of the Hahn-Banach type theorems, emphasizing the topological versions of these applications.
\end{abstract}

Keywords: Hahn-Banach type theorems; Markov moment problem; sandwich theorem; finite-simplicial set; isotone convex operator; necessary and sufficient conditions

\section{Introduction}

We recall the classical formulation of the moment problem, under the terms of T. Stieltjes, given in 1894-1895 (see the basic book of N.I. Akhiezer [1] for details): find the repartition of the positive mass on the nonnegative semi-axis, if the moments of arbitrary orders $k(k=0,1,2, \ldots)$ are given. Precisely, in the Stieltjes moment problem, a sequence of real numbers $\left(S_{k}\right)_{k \geq 0}$ is given and one looks for a nondecreasing real function $\sigma(t)(t \geq 0)$, which verifies the moment conditions:

$$
\int_{0}^{\infty} t^{k} d \sigma=s_{k}(k=0,1,2, \ldots)
$$

This is a one dimensional moment problem, on an unbounded interval. Namely, it is an interpolation problem with the constraint on the positivity of the measure $d \sigma$. The numbers $S_{k}, k \in \mathbb{N}=\{0,1,2, \ldots\}$ are called the moments of the measure $d \sigma$ Existence, uniqueness and construction of the solution are studied. The present work concerns firstly the existence problem. The connection with the positive polynomials and extensions of linear positive functional and/or operators is quite clear. Namely, if one denotes by $\varphi_{j}$, $\varphi_{j}(t):=t^{j}, j \in \mathbb{N}, t \in \mathbb{R}_{+}:=[0, \infty), \mathcal{P}$ the vector space of polynomials with real coefficients and

$$
T_{0}: \mathcal{P} \rightarrow \mathbb{R}, T_{0}\left(\sum_{j \in J_{0}} \alpha_{j} \varphi_{j}\right):=\sum_{j \in J_{0}} \alpha_{j} S_{j},
$$

where $J_{0} \subset \mathbb{N}:=\{0,1,2, \ldots\}$ is a finite subset, then the moment conditions $T_{0}\left(\varphi_{j}\right)=s_{j}, j \in \mathbb{N}$ are clearly verified. It remains to check whether the linear form $T_{0}$ has nonnegative values at nonnegative 
polynomials. If this last condition is also accomplished, then one looks for the existence of a linear positive extension $T$ of $T_{0}$ to a larger ordered function space $E$ which contains both $\mathcal{P}$ and the space of continuous compactly supported functions, then representing $T$ by means of a positive regular Borel measure $\mu$ on $[0, \infty)$, via Riesz representation theorem. Alternately one can apply directly Haviland theorem. Usually, $E$ is an ordered Banach space or even a Banach lattice. For Markov moment problem, besides the positivity of the linear solution $T$, the condition $T \leq P$ on $E$ is required, where $P: E \rightarrow \mathbb{R}$ is a given convex (eventually continuous) functional. In most of the cases, $P$ is a continuous sublinear functional, which controls the norm of the linear positive solution $T$. Similar results make sense and can be proved for the multidimensional moment problem. If an interval (for example $[a, b], \mathbb{R}$, or $\mathbb{R}_{+}:=[0, \infty)$ ) is replaced by a closed subset of $\mathbb{R}^{n}, n \geq 2$, we have a multidimensional moment problem. Passing to the multidimensional real Stieltjes moment problem, let us denote

$$
\varphi_{j}(t)=t^{j}=t_{1}^{j_{1}} \cdots t_{n}^{j_{n}}, j=\left(j_{1}, \ldots, j_{n}\right) \in \mathbb{N}^{n}, t=\left(t_{1}, \ldots, t_{n}\right) \in \mathbb{R}_{+}^{n}, n \in \mathbb{N}, n \geq 2
$$

If a sequence $\left(y_{j}\right)_{j \in \mathbb{N}^{n}}$ is given, one studies the existence, uniqueness and construction of a linear positive form $T$ defined on a Banach function spaces containing polynomials and continuous compactly supported functions, such that the moment conditions

$$
T(\varphi)=y_{j}, j \in \mathbb{N}^{n}
$$

are satisfied. Usually, the positive linear form $T$ can be represented by means of a positive regular Borel measure on $\mathbb{R}_{+}^{n}$. When an upper constraint on the solution $T$ is required too, we have a Markov moment problem. This requirement is formulated as $T$ being dominated by a convex functional, which might be a norm or only a sublinear functional, whose role is to control the continuity and the norm of the solution. All these aspects motivate the study of the abstract moment problem, from which we deduce results for the classical Markov moment problem by means of additional information (usually involving nonnegative polynomials, sums of squares of polynomials, quadratic forms, measure theory, etc.). Clearly, the classical (Stieltjes) moment problem is an extension problem for linear functionals, from the subspace of polynomials to a function space which contains both polynomial as well as the continuous compactly supported real functions on $\mathbb{R}_{+}^{n}$. From linear functional solutions, many authors considered linear operator solutions. Of course, in this case the moments $y_{j}, j \in \mathbb{N}^{n}$ are elements of an ordered complete vector space $Y$ (usually $Y$ is an order complete Banach space (or an order complete Banach lattice)). The order completeness is necessary to apply Hahn-Banach type results for operators defined on polynomials and having $Y$ as codomain. Various aspects of the classical moment problem have been studied (see the References). Basic monographs on the moment problem can be found in references [1-3]. General background can be found in chapters or paragraphs from monographs [4-8]. The present paper is directly related to references [9-15] and partially related to articles [16-20]. The case of multidimensional moment problem on compact semi-algebraic subsets in $\mathbb{R}^{n}$ was intensively studied. The analytic form of positive polynomials on special closed unbounded finite dimensional subsets is crucial in solving classical moment problems on such subsets (see [21] for the expression of nonnegative polynomials on a strip, in terms of sums of squares). All the references [22-46] are more or less linked to various aspects of the moment problem and its relationship with other fields of analysis. Clearly, the classical moment problem is related to the form of positive polynomials on the involved closed subset of $\mathbb{R}^{n}$. As it is well-known, there exists nonnegative polynomials on the entire space $\mathbb{R}^{n}, n \geq 2$, which are not sums of squares of polynomials (cf. [23]), unlike the case $n=1$. Connections with fixed point theory are emphasized in $[24,25]$, while related results on operator theory are pointed out in $[3,27,32,34,35]$. Uniqueness/nonuniqueness of the solutions of some classical moment problems are studied in [3,29-31]. Optimization related to Markov moment problem, and, on the other hand, construction of the solution is emphasized as well in some of the references. It is worth noticing that our References are far from being complete. For the 
reader's convenience, we include the following recent main research publications on Hahn-Banach theorem, sandwich theorem and their applications [47-51]. Going further to the aims of the present work, we start by recalling a result on the abstract moment problem, which follows from more general theorems previously published. This statement (see Theorem 3 below) was applied in [13] to deduce a sandwich type result of intercalating an affine functional $h: X \rightarrow \mathbb{R}$ between a convex functional $f: X \rightarrow \mathbb{R}$ and a concave functional $g: X \rightarrow \mathbb{R}, f \leq g$, where $X$ is a finite simplicial subset of a real vector space $E$ (see Section 3 below for the definition and examples). Here the novelty is that a finite-simplicial set $X$ may be unbounded in any locally convex topology on $E$. In the present paper, we prove a topological version of this sandwich result. This is the first aim of this work. Some other related results are recalled or respectively proved. It is worth noticing that, unlike Hahn-Banach theorems, here the dominating functional $g$ is concave, while the minorating functional $f$ is convex. A previous such sandwich result for real functions defined on a (compact) Choquet simplex $X$ is recalled in [6] and in Section 3.1 below. A topological version of it is also proved. Section 3.2 is devoted to a direct sharp proof of a generalization of Hahn-Banach theorem, motivated by solving Markov moment problems and by characterization of isotonicity of convex operators over convex cones [14,15] (recall that an operator $P$ acting between two ordered vector spaces $E, F$ is called isotone on the cone $C \subset E$ if

$$
x_{1}, x_{2} \in C, x_{1} \leq x_{2} \Longrightarrow P\left(x_{1}\right) \leq P\left(x_{2}\right) .
$$

This is the second aim of the present paper. Finally, extending inequalities from a small subset to the entire positive cone of the domain space is the third aim of this article. This is realized by means of Krein-Milman and, respectively, Carathéodory theorem, both of them being consequences of the geometric form of Hahn-Banach theorem. Thus, we have four directions of application of Hahn-Banach type results: Markov moment problem, proving sandwich results over finite-simplicial sets, characterizing isotonicity of convex operators over convex cones and extending inequalities via Krein-Milman type theorems.

\section{Materials and Methods}

The basic methods used in this work are:

(1) Extension of linear operators such that the extension is dominated by a convex operator and minorated by a concave operator (see [9-11]). The convex and concave operators, respectively, are defined on arbitrary convex subsets $A, B$ of the domain space $E$. An important particular case is that when $A$ or $B$ (or both) is (are) equal to the positive cone $E_{+}$. The codomain space is always order complete, in order to allow applying Hahn-Banach results.

(2) Characterizing existence of solutions for Markov moment problem in terms of the given moments $y_{j}, j \in J$.

(3) From one of the results mentioned at point (2), proving a sandwich result over a finite simplicial set. Adding the topological version of this result.

(4) Giving a sharp direct proof for a generalization of Hahn-Banach theorem and applying it to a very recent result on isotonicity of a convex operator over a convex cone (see $[14,15])$. On the other hand, a variant of this generalized Hahn-Banach result was frequently applied to the Markov moment problem. It allows controlling the norm of the solution $T$ (see also Remark 5 below).

(5) Using Krein-Milman and Caratheodory's theorems in order to extend inequalities from a smaller set to the entire positive cone of the domain. Applying the notion of a quasiconvex operator [5].

\section{Results}

\subsection{Simplexes, Finite-Simplicial Sets and Sandwich Theorems over Such Subsets}

The aim of this subsection is to prove topological versions for known sandwich results $[6,13]$. We start by a preliminary lemma. Recall that a base $B$ of a convex cone $C$ contained in a vector space $E$ 
is a set of the form $B=C \cap H$, where $H$ is a hyperplane which misses the origin, defined with the aid of a strictly positive linear functional

$$
\varphi: E \rightarrow \mathbb{R}(\varphi(x)>0 \text { for all } x \in C, x \neq 0)
$$

If such a functional does exist, then choose any real $\beta>0$ and define

$$
H=\{x \in E ; \varphi(x)=\beta\}
$$

Then for any $x \in C, x \neq 0$, there exists a unique $b \in B=H \cap C$ and a unique real number $\alpha>0$ such that

$$
x=\alpha b
$$

Indeed, if $b=\beta \frac{x}{\varphi(x)}$, then $b \in B$ and $x=\frac{\varphi(x)}{\beta} b=a b$.

For the next result we will restrict ourselves to the Banach lattice setting, since the topological properties involved are important. In this respect, the hyperplane $H$ and the positive cone $E_{+}$are closed subsets ( $\varphi$ is continuous).

Lemma 1. Let E, F be Banach lattices. Assume that the positive cone $E_{+}$has a base

$$
B=H \cap E_{+},
$$

where $H$ is a closed hyperplane missing the origin, as mentioned above. Let $p,-q: B \rightarrow F$ be convex continuous bounded operators on $B$ and $\varphi: B \rightarrow F$ an affine continuous bounded operator. Define

$$
\Phi, \Psi: E_{+} \rightarrow F, \Phi(\lambda b)=\lambda p(b), \Psi(\lambda b)=\lambda q(b), b \in B, \lambda \in \mathbb{R}_{+}
$$

Then $\Phi$ is a sublinear continuous extension of $p, \Psi$ is supralinear, continuous and extends $q$, while

$$
T(\lambda b)=\lambda \varphi(b)
$$

defines an additive positively homogeneous operator on $E_{+}$, which can be further extended to a linear continuous operator $T$ from $E$ to $F$. In addition, we have

$$
p \leq \varphi \leq q \text { on } B \text { if and only if } \Phi \leq T \leq \Psi \text { on } E_{+}
$$

Proof. By definition, $\Phi, \Psi, T$ extend $p, q, \varphi$ respectively, from $B$ to $E_{+}$. To prove that $\Phi$ is subadditive, let $x_{1}=\lambda_{1} b_{1}, x_{2}=\lambda_{2} b_{2}$ be two elements in the positive cone $E_{+}$, where

$$
b_{j} \in B, \lambda_{j} \in[0, \infty), j=1,2, \lambda_{1}+\lambda_{2}>0
$$

Then

$$
\begin{gathered}
\Phi\left(x_{1}+x_{2}\right)=\Phi\left(\left(\lambda_{1}+\lambda_{2}\right)\left(\frac{\lambda_{1}}{\lambda_{1}+\lambda_{2}} b_{1}+\frac{\lambda_{2}}{\lambda_{1}+\lambda_{2}} b_{2}\right)\right):= \\
\left(\lambda_{1}+\lambda_{2}\right) p\left(\frac{\lambda_{1}}{\lambda_{1}+\lambda_{2}} b_{1}+\frac{\lambda_{2}}{\lambda_{1}+\lambda_{2}} b_{2}\right) \leq \\
\left(\lambda_{1}+\lambda_{2}\right)\left(\frac{\lambda_{1}}{\lambda_{1}+\lambda_{2}} p\left(b_{1}\right)+\frac{\lambda_{2}}{\lambda_{1}+\lambda_{2}} p\left(b_{2}\right)\right)= \\
\lambda_{1} p\left(b_{1}\right)+\lambda_{2} p\left(b_{2}\right)=\Phi\left(x_{1}\right)+\Phi\left(x_{2}\right)
\end{gathered}
$$


Next we prove that $\Phi$ is positively homogeneous: if $x=\lambda b, b \in B, \lambda \in(0, \infty), \alpha \in(0, \infty)$, then

$$
\Phi(\alpha x)=\Phi((\alpha \lambda) b)=(\alpha \lambda) p(b)=\alpha(\lambda p(b))=\alpha \Phi(x)
$$

Thus, $\Phi$ is sublinear, $\Psi$ is supralinear and $T$ is additive and positively homogeneous on the positive cone of $E$ since $\varphi$ is assumed to be simultaneous convex and concave on $B$.

Extend $T$ to the space

$$
E=E_{+}-E_{+} \text {by } T\left(x_{1}-x_{2}\right):=T\left(x_{1}\right)-T\left(x_{2}\right), \text { where } x_{j} \in E_{+}, j=1,2
$$

The definition makes sense, since it does not depend on decomposing $x$ as a difference of two arbitrary elements from the positive cone $E_{+}$. Indeed, if $x_{1}-x_{2}=y_{1}-y_{2}$, then

$$
x_{1}+y_{2}=x_{2}+y_{1}, x_{j}, y_{j} \in E_{+}, j=1,2
$$

Now additivity of $T$ on $E_{+}$yields

$$
T\left(x_{1}\right)+T\left(y_{2}\right)=T\left(x_{2}\right)+T\left(y_{1}\right) \Leftrightarrow T\left(x_{1}\right)-T\left(x_{2}\right)=T\left(y_{1}\right)-T\left(y_{2}\right)
$$

For each nonnegative scalar $\lambda$, clearly it results

$$
T(\lambda x)=T\left(\lambda x_{1}\right)-T\left(\lambda x_{2}\right)=\lambda\left(T\left(x_{1}\right)-T\left(x_{2}\right)\right)=\lambda T(x), x_{j} \in E_{+}, j=1,2
$$

On the other hand, according to definition from above, we infer that

$$
T(-x)=T\left(-\left(x_{1}-x_{2}\right)\right)=T\left(x_{2}\right)-T\left(x_{1}\right)=-\left(T\left(x_{1}\right)-T\left(x_{2}\right)\right)=-T(x)
$$

If $\lambda<0$, then $\lambda=-\rho$, where $\rho=-\lambda>0$. From the preceding remark, we derive

$$
T(\lambda x)=T(-\rho x)=\rho T(-x)=-\rho T(x)=\lambda T(x)
$$

To finish the proof, we only have to prove the assertions on the continuity. If $\left(x_{n}\right)_{n \geq 1}$ is a sequence of elements from $E_{+}$, such that

$$
x_{n}=\lambda_{n} b_{n} \rightarrow x=\lambda b, b_{n}, b \in B, \lambda_{n}, \lambda>0
$$

then the following relations hold true

$$
\varphi\left(x_{n}\right)=\lambda_{n} \varphi\left(b_{n}\right)=\lambda_{n} \beta \rightarrow \varphi(x)=\lambda \beta,
$$

Since $\varphi$ is continuous, being a positive linear functional on the Banach lattice $E$. Thus, $\lambda_{n} \rightarrow \lambda$.

These relations lead to

$$
b_{n}=\frac{1}{\lambda_{n}} x_{n} \rightarrow \frac{1}{\lambda} x=b
$$

Due to the continuity of $p$ on $B$, it results $p\left(b_{n}\right) \rightarrow p(b)$, which further implies

$$
\Phi\left(x_{n}\right)=\lambda_{n} p\left(b_{n}\right) \rightarrow \lambda p(b)=\Phi(x)
$$

A special case is $x=0$, when

$$
\begin{gathered}
x_{n}=\lambda_{n} b_{n} \rightarrow 0 \Rightarrow \lambda_{n} \beta=\varphi\left(x_{n}\right) \rightarrow 0 \Rightarrow \lambda_{n} \rightarrow 0 \Rightarrow \\
\Phi\left(x_{n}\right)=\lambda_{n} p\left(b_{n}\right) \rightarrow 0,
\end{gathered}
$$


since $p$ is bounded on $B$ by hypothesis. Thus, the continuity of $\Phi, \Psi$ and $T$ on $E_{+}$is proved. We only have to show that the linear extension of $T$ to the whole space $E$ is continuous, where this extension has been denoted by $T$ too. Clearly, the continuity at the origin will be sufficient. The following implications hold true

$$
x_{n} \rightarrow 0 \Rightarrow x_{n}^{+} \rightarrow 0 \Rightarrow T\left(x_{n}^{+}\right) \rightarrow 0
$$

Similarly, $T\left(x_{n}^{-}\right) \rightarrow 0$, hence $T\left(x_{n}\right)=T\left(x_{n}^{+}\right)-T\left(x_{n}^{-}\right) \rightarrow 0$. Finally, observe that

$$
\begin{gathered}
p \leq h \leq q \text { on } B \Leftrightarrow \lambda p(b)=\Phi(\lambda b) \leq \lambda h(b)=T(\lambda b) \leq \lambda q(b)=\Psi(\lambda b), \lambda>0, b \in B \Leftrightarrow \\
\Phi(x) \leq T(x) \leq \Psi(x), x \in E_{+}
\end{gathered}
$$

The proof is complete.

Next, we recall the definition of a simplex in an infinite dimensional locally convex space $E$, and we emphasize one of its main properties. If $C$ is a convex cone in $E, C$ has a base $B$ and the order relation defined by $C$ is laticial on $E_{1}=C-C$, then $B$ is called a Choquet simplex. Usually, a simplex is assumed to be compact. For more information on simplexes see [6]. Next we recall the statement of D.A. Edwards' separation theorem (Theorem 16.7 [6]).

Lemma 2. (Edwards). If $f$ and $-g$ are convex upper semicontinuous real valued functions on a simplex $B$ contained in a locally convex space, with $f \leq g$, then there exists a continuous affine function $h$ on $B$ such that $f \leq h \leq g$.

The next result is a topological version of Lemma 2.

Theorem 1. Let E be a locally convex space, $C$ a pointed convex cone in E which has a simplex $B$ as a base. Assume that the trace of the topology of $E$ on $E_{1}=C-C$ is locally solid with respect to the order relation defined by C. Let $\Phi: C \rightarrow \mathbb{R}$ be a continuous sublinear functional, $\Psi: C \rightarrow \mathbb{R}$ a continuous supralinear functional such that $\Phi(e)=\Psi(e)$ for all $e \in \operatorname{Ex}(B)$. Then there exists a unique continuous linear functional $T: E_{1} \rightarrow \mathbb{R}$ such that

$$
\Phi(x) \leq T(x) \leq \Psi(x) \forall x \in C
$$

Proof. Let $f:=\left.\Phi\right|_{B}, g:=\left.\Psi\right|_{B}$. Then $f$ is convex and continuous, $g$ is concave and continuous, and $f \leq g$ on $B(f-g$ is continuous and convex, vanishing on $E x(B)$ by hypothesis; it results $(f-g)(x) \leq 0$ for all $x \in \operatorname{co}(\operatorname{Ex}(B))$ and via continuity, $(f-g)(x) \leq 0$ for all $x \in c l(\operatorname{co}(\operatorname{Ex}(B)))=B$, where the last equality is given by Krein-Milman theorem). Since $B$ is a simplex, application of Lemma 2 leads to the existence of a continuous affine function $h: B \rightarrow \mathbb{R}$, such that $f \leq h \leq g$. According to the first part of the proof of Lemma 1 (which does not use any topological notions), $h$ has a unique linear extension, say $T$, to $E_{1}=C-C$, such that (Lemma 1 ) holds true. The next step is to prove the continuity of $T$ on $E_{1}$, which is equivalent to its continuity at the origin. Let $\left(x_{\delta}\right)_{\delta \in \Delta}$ be a generalized sequence in $E_{1}$, such that $x_{\delta} \rightarrow 0$. Consider the sequences $\left(x_{\delta}^{+}\right)_{\delta \in \Delta^{\prime}}\left(x_{\delta}^{-}\right)_{\delta \in \Delta}$ in $C$. According to the assumptions on the topology on $E_{1}$, also using the continuity at the origin of $\Phi, \Psi$, and (3) as well, we infer that

$$
\begin{gathered}
x_{\delta} \rightarrow 0 \Rightarrow\left(x_{\delta}^{+} \rightarrow 0, x_{\delta}^{-} \rightarrow 0\right) \Rightarrow \\
\left(\Phi\left(x_{\delta}^{+}\right) \rightarrow 0, \Psi\left(x_{\delta}^{+}\right) \rightarrow 0, \Phi\left(x_{\delta}^{+}\right) \leq T\left(x_{\delta}^{+}\right) \leq \Psi\left(x_{\delta}^{+}\right)\right) \Rightarrow T\left(x_{\delta}^{+}\right) \rightarrow 0
\end{gathered}
$$

Similarly, $T\left(x_{\delta}^{-}\right) \rightarrow 0$, so that

$$
T\left(x_{\delta}\right)=T\left(x_{\delta}^{+}\right)-T\left(x_{\delta}^{-}\right) \rightarrow 0
$$


Hence $T$ is continuous. To finish the proof, we only have to show the uniqueness of $T$ with the properties in the statement. Let $T_{1}$ be a linear continuous functional on $E_{1}$ such that (3) holds for $T_{1}$ instead of $T$. Let $h_{1}$ be the restriction of $T_{1}$ to the simplex $B$. Then $h_{1}$ is continuous and affine on $B$. Moreover, we have

$$
\begin{gathered}
h_{1}(e)=T_{1}(e) \in[\Phi(e), \Psi(e)]=\{T(e)\}=\{h(e)\} \forall e \in E x(B) \Rightarrow \\
h_{1}(e)=\{h(e)\} \forall e \in \operatorname{Ex}(B)
\end{gathered}
$$

This further yield $h_{1}(x)=h(x)$ for all $x \in \operatorname{co}(E x(B))$ (via the property of being affine for $h, h_{1}$ ). Now also using the continuity of the two involved functions, we derive

$$
h_{1}(x)=h(x) \text { for all } x \in \operatorname{cl}(\operatorname{co}(E x(B)))=B,
$$

where the last equality is a consequence of Krein-Milman theorem. Thus, $\left.T_{1}\right|_{B}=\left.T\right|_{B}$, which means $T_{1}=T$ on $E_{1}$. This ends the proof.

Remark 1. Let $E=l_{1}$ and $F$ an arbitrary Banach space endowed with a linear order relation such that the positive cone $F_{+}$is closed. Let $\left\{e_{n}\right\}_{n=1}^{\infty}$ be the canonical base of $E$ and $\left\{y_{n}\right\}_{n=1}^{\infty}$ a bounded sequence in $F$. Assume that $\Phi,-\Psi: E_{+} \rightarrow F$ are given sublinear continuous operators such that $\Phi\left(e_{n}\right)=\Psi\left(e_{n}\right)$ for all natural numbers $n \geq 1$. Then there exists a unique bounded linear operator $T \in \mathcal{L}(E, F)$ such that $\Phi \leq\left. T\right|_{E_{+}} \leq \Psi$. Indeed, if we denote

$$
y_{n}=\Phi\left(e_{n}\right), n \geq 1 \text {. }
$$

and define

$$
T(x)=\sum_{n=1}^{\infty} x_{n} y_{n}, x=\left(x_{n}\right)_{n \geq 1} \in E,
$$

then the series on the right hand side is absolutely convergent in the Banach space F for each fixed element $x \in E$. Thus, (4) defines a linear operator, which is continuous, with

$$
\|T\|=\sup _{n \geq 1}\left\|y_{n}\right\|<\infty
$$

From (4), also using the hypothesis, it results $T\left(e_{n}\right)=y_{n}=\Phi\left(e_{n}\right)=\Psi\left(e_{n}\right), n \geq 1$. These last equalities and continuity of all involved operators, as well as sublinearity of $\Phi,-\Psi$ lead to

$$
\Phi(x) \leq T(x) \leq \Psi(x)
$$

for all $x \in E_{+}$. It is possible that such examples could be adapted for more general spaces $E$.

In the end of this section, we recall that related sandwich theorems hold on the finite-simplicial sets, as discussed in [13]. A convex subset $X$ of the vector space $E$ is called finite-simplicial if for any finite dimensional convex compact subset $K \subset X$, there exists a finite dimensional simplex $S$ such that $K \subset S \subset X$. The novelty here is that $X$ is not supposed to be bounded in a locally convex topology on $E$. Here are a few examples.

(1) In $\mathbb{R}^{n}, n \geq 2$, any convex cone $X$ having a base that is a simplex (the corresponding order relation is laticial) is an unbounded finite simplicial set.

(2) In $\mathbb{R}^{n}, n \geq 2$, for each $p \in(1, \infty)$, the convex cone $X$ defined by

$$
X=\left\{\left(x_{1}, \ldots, x_{n}\right) ; x_{n} \geq\left(\sum_{j=1}^{n-1}\left|x_{j}\right|^{p}\right)^{1 / p}\right\}
$$


has a compact base, but $X$ is not finite-simplicial.

(3) Let $E$ be an arbitrary infinite or finite dimensional vector space (of dimension $\geq 2$ ), $T: E \rightarrow \mathbb{R}$ a non-null linear functional and $\alpha \in \mathbb{R}$. Then the sets $X_{1}=\{x ; T(x) \geq \alpha\}, X_{2}=\{x ; T(x) \leq \alpha\}$ are finite-simplicial.

(4) Let $E, T$ be as in Example (3), $\alpha, \beta$ two real numbers such that $\alpha<\beta$. The set

$$
X=\{x ; \alpha \leq T(x) \leq \beta\}
$$

is not finite-simplicial. From the last two examples, we easily infer that generally the intersection of two finite-simplicial sets is not finite-simplicial.

The following sandwich type result holds true (cf. [13]).

Theorem 2. Let $E$ be an arbitrary vector space, $X$ a finite-simplicial subset, $f: X \rightarrow \mathbb{R}$ a convex functional, $g: X \rightarrow \mathbb{R}$ a concave functional such that $f \leq g$ on $X$. Then there exists an affine functional $h: X \rightarrow \mathbb{R}$ such that $f \leq h \leq g$.

The proof is based on the following theorem first published in [11] and recently generalized in [46].

Theorem 3. Let $E$ be an ordered vector space, $F$ an order complete vector lattice, $\left\{\varphi_{j}\right\}_{j \in J} \subset E,\left\{y_{j}\right\}_{j \in J} \subset F$ given arbitrary families, $T_{1}, T_{2} \in L(E, F)$ two linear operators. The following statements are equivalent:

(a) There is a linear operator $T \in L(E, F)$ such that

$$
T_{1}(x) \leq T(x) \leq T_{2}(x) \forall x \in E_{+}, T\left(\varphi_{j}\right)=y_{j} \forall j \in J ;
$$

(b) For any finite subset $J_{0} \subset J$ and any $\left\{\lambda_{j} ; j \in J_{0}\right\} \subset \mathbb{R}$, the following implication holds true

$$
\left(\sum_{j \in J_{0}} \lambda_{j} \varphi_{j}=\psi_{2}-\psi_{1}, \psi_{1}, \psi_{2} \in E_{+}\right) \Longrightarrow \sum_{j \in J_{0}} \lambda_{j} y_{j} \leq T_{2}\left(\psi_{2}\right)-T_{1}\left(\psi_{1}\right)
$$

If $E$ is a vector lattice, then assertions ( $a$ ) and (b) are equivalent to (c), where

(c) $T_{1}(w) \leq T_{2}(w)$ for all $w \in E_{+}$and for any finite subset $J_{0} \subset J$ and $\forall\left\{\lambda_{j} ; j \in J_{0}\right\} \subset \mathbb{R}$, we have

$$
\sum_{j \in J_{0}} \lambda_{j} y_{j} \leq T_{2}\left(\left(\sum_{j \in J_{0}} \lambda_{j} \varphi_{j}\right)^{+}\right)-T_{1}\left(\left(\sum_{j \in J_{0}} \lambda_{j} \varphi_{j}\right)^{-}\right)
$$

The next result is new, representing a topological version of Theorem 2.

Theorem 4. Let E be an ordered Banach space. Assume that $E_{+}$is finite-simplicial and there exists

$$
x_{0} \in E_{+}
$$

such that $\mathrm{E}_{+}-\mathrm{x}_{0}$ contains a balanced, absorbing, convex subset. Let

$$
f,-g: E_{+} \rightarrow \mathbb{R}
$$

be convex continuous functions such that $f \leq g$ Assume also that $f(\mathbf{0})=g(\mathbf{0})=0$. Then there exists a continuous linear form $S: E \rightarrow \mathbb{R}$ such that $f \leq S \leq g$ on $E_{+}$. 
Proof. According to Theorem 2, there exists an affine functional $h: E_{+} \rightarrow \mathbb{R}$ such that

$$
f \leq h \leq g \text { on } E_{+}
$$

Using also the hypothesis, we infer that

$$
0=f(\mathbf{0}) \leq h(\mathbf{0}) \leq g(\mathbf{0})=0,
$$

hence $h(\mathbf{0})=0$. On the other hand, since $E_{+}-x_{0}$ is convex, absorbing and balanced, it is a neighborhood of $\mathbf{0}$ in the finest locally convex topology on $E$ (see [7]). Recall that in this topology, any convex balanced absorbing subset is a neighborhood of $\mathbf{0}$ (this is the topology generated by the family of all seminorms on $E$ ). Thus, there exists a convex open neighborhood $V$ of $\mathbf{0}$ in this topology, such that

$$
D=x_{0}+V \subset E_{+}
$$

In particular, the interior $i n\left(E_{+}\right)$of $E_{+}$with respect to the finest locally convex topology on $E$ is not empty. Denote this interior by $C$. Since $h$ is convex and concave on the convex open subset $C$ (in the finest locally convex topology on $E$ ) and $x_{0} \in C$, the subdifferentials of $h$ and $-h$ at $x_{0}$ are nonempty. This means that there exist linear functionals $T$ and $U$ on $E$ such that

$$
T(x)-T\left(x_{0}\right) \leq h(x)-h\left(x_{0}\right) \leq U(x)-U\left(x_{0}\right)
$$

for all $x \in C$. Since clearly any linear form on $E$ is continuous with respect to the finest locally convex topology, from the last two inequalities we infer that $h$ is continuous at $x_{0}$. Repeating this argument for any $x_{0} \in C$, we infer that $h$ is continuous on $C$ with respect to the topology under attention. As a consequence, the subsets

$$
A=\{(x, r) \in C \times \mathbb{R} ; h(x)<r\}, B=\{(x, r) \in C \times \mathbb{R} ; h(x)>r\}
$$

are open convex disjoint subsets in $C \times \mathbb{R}$ endowed with the product topology. By separation theorem, there exists a hyperplane $H \subset E \times \mathbb{R}$, separating (strictly) the subsets $A$ and $B$. So, there is a not null linear form $L$ on $E \times \mathbb{R}$ and $\alpha \in \mathbb{R}$ such that

$$
\begin{gathered}
H=\{(x, r) \in E \times \mathbb{R} ; L(x, r)=\alpha\}, A \subset\{(x, r) \in C \times \mathbb{R} ; L(x, r)<\alpha\}, \\
B \subset\{(x, r) \in C \times \mathbb{R} ; L(x, r)>\alpha\}
\end{gathered}
$$

All the topological notions in the sequel refer to the finest locally convex topology, unless another specification is mentioned. Since $L$ is linear, it is continuous on $E \times \mathbb{R}$. For $\left(x, r_{1}\right) \in A$, we have

$$
L(x, 0)+r_{1} L(\mathbf{0}, 1)<\alpha,
$$

while for any $\left(x, r_{2}\right) \in B$, it results

$$
L(x, 0)+r_{2} L(\mathbf{0}, 1)>\alpha
$$

From these last two inequalities written for a $x \in C$ and $r_{1} \neq r_{2}$, we infer that

$$
L(x, 0)+r_{1} L(\mathbf{0}, 1)<\alpha<L(x, 0)+r_{2} L(\mathbf{0}, 1)
$$

that implies $\left(r_{2}-r_{1}\right) L(0,1)>0$. In particular, it results $L(\mathbf{0}, 1) \neq 0$. Going back to the definitions of the subsets $A$ and $B$, observe that for any $x \in C$ and any $\varepsilon>0$ the element $(x, h(x)+\varepsilon) \in A$, while $(x, h(x)-\varepsilon) \in B$. This remark yields

$$
L(x, 0)+(h(x)+\epsilon) L(\mathbf{0}, 1)<\alpha, L(x, 0)+(h(x)-\epsilon) L(\mathbf{0}, 1)>\alpha
$$


Passing to the limit as $\varepsilon \rightarrow 0$, one obtains

$$
L(x, 0)+h(x) L(\mathbf{0}, 1)=\alpha
$$

for all $x \in C$. This may be written as

$$
h(x)=\frac{\alpha}{L(\mathbf{0}, 1)}-\frac{L(x, 0)}{L(\mathbf{0}, 1)}
$$

Hence $h$ is the restriction to $C=\operatorname{in}\left(E_{+}\right)$of the linear form $S$ defined on $E$ by

$$
S(x)=-\frac{L(x, 0)}{L(\mathbf{0}, 1)}
$$

then adding the constant $\frac{\alpha}{L(\mathbf{0}, 1)}$. Recall now that we have already proved that $h(\mathbf{0})=0$, that yields $\alpha=0$, so that $h$ is the restriction to $C$ of the linear form $S$. On the other hand, by our hypothesis, $f$ and $g$ are assumed to be continuous with respect to the norm topology. In particular, they are continuous with respect to the finest locally convex topology and $S$ has this property as well. We also have

$$
\left.f\right|_{C} \leq\left. S\right|_{C} \leq\left. g\right|_{C}
$$

and $C=\operatorname{in}\left(E_{+}\right)$is dense in $E_{+}$. From the continuity of the three involved functionals, the inequalities written above are extended to the entire positive cone $E_{+}$. To finish the proof, we must show that $S$ is continuous with respect to the norm topology, that is equivalent to its continuity at $\mathbf{0}$. From now on, only the norm topology is involved. Let $x_{n} \rightarrow \mathbf{0}$. As it is known, the ordered Banach space $E$ can be renormed by means of an equivalent norm, such that it becomes a regularly ordered Banach space. In such a space for any sequence $x_{n} \rightarrow \mathbf{0}$, there exists sequences $\left(u_{n}\right)_{n},\left(v_{n}\right)_{n}, u_{n}, v_{n} \in E_{+}, x_{n}=u_{n}-v_{n}$ for all $n \in \mathbb{N}$, such that $u_{n} \rightarrow \mathbf{0}, v_{n} \rightarrow \mathbf{0}$. It results

$$
0=f(\mathbf{0}) \leftarrow f\left(u_{n}\right) \leq S\left(u_{n}\right) \leq g\left(u_{n}\right) \rightarrow g(\mathbf{0})=0
$$

which obviously implies $S\left(u_{n}\right) \rightarrow 0$. Similarly, $S\left(v_{n}\right) \rightarrow 0$, so that

$$
S\left(x_{n}\right)=S\left(u_{n}\right)-S\left(v_{n}\right) \rightarrow 0
$$

This concludes the proof.

\subsection{A Direct Proof for a Generalization of Hahn-Banach Theorem and Its Motivation}

The following theorem has been recently applied in [14] to a characterization of isotonicity of a continuous convex operator over a convex cone in terms of its subgradients (see [14], Theorem 6). It can be obtained from more general results published in $[9,10]$. The proof of these very general results is quite long and technical. Therefore, it is preferable to have a direct proof, using only Zorn lemma and appropriate inequalities.

Theorem 5. (Theorem 3 [14]). Let $E$ be an ordered vector space, $F$ an order complete vector space, $H \subset E a$ vector subspace, $T_{1}: H \rightarrow F$ a linear operator, $\Phi: E_{+} \rightarrow F$ a convex operator. The following statements are equivalent:

(a) There exists a positive linear extension $T: E \rightarrow F$ of $T_{1}$ such that $\left.T\right|_{E_{+}} \leq \Phi$;

(b) We have $T_{1}(h) \leq \Phi(x)$ for all $(h, x) \in H \times E_{+}$such that $h \leq x$. 
Proof. The implication $(a) \Longrightarrow(b)$ is obvious; indeed, we have

$$
T_{1}(h)=T(h) \leq T(x) \leq \Phi(x)
$$

$(h, x) \in H \times E_{+}$such that $h \leq x$, thanks to the positivity of $T$, also using the property $T(x) \leq \Phi(x)$ for all $x \in E_{+}$. To prove the converse, let $\Phi$ be an arbitrary convex operator over $E_{+}$, verifying the conditions mentioned at (b). We are going to apply Zorn lemma to the set $\mathcal{L}$ of all pairs $\left(K, T_{K}\right)$, where $K$ is a vector subspace of $E, H \subset K, T_{K}: K \rightarrow F$ is a linear operator such that $\left.T_{K}\right|_{H}=T_{1}$ and $T_{K}(h) \leq \Phi(x)$ for all $h \in K$ and $x \in E_{+}$such that $h \leq x$. The set $\mathcal{L}$ contains the pair $\left(H, T_{1}\right)$ and is inductively ordered by the order relation

$$
\left(K, T_{K}\right) \ll\left(L, T_{L}\right) \Leftrightarrow K \subset L,\left.T_{L}\right|_{K}=T_{K}
$$

According to Zorn's Lemma, there exists a maximal pair $\left(H_{M}, T_{H_{M}}\right) \in \mathcal{L}$. Our aim is to prove that $H_{M}=E$. Assuming this is achieved, and taking $T:=T_{H_{M}}$, we have $T(h) \leq \Phi(x)$ for all $h \in H$ and $x \in E_{+}$such that $h \leq x$. Application of this inequality for $h=-n y, y \in E_{+}, n \in \mathbb{N}, x=0$, yields

$$
n T(-y)=T(-n y) \leq \Phi(\mathbf{0}), n \in \mathbb{N}
$$

Since any order complete vector space is Archimedean, it results $T(-y) \leq \mathbf{0}, y \in E_{+}$, that is the positivity of $T$. Additionally, taking $h=x \in E_{+}$, one obtains $T(x) \leq \Phi(x)$ and $T$ will be the expected positive extension of $T_{1},\left.T\right|_{E_{+}} \leq \Phi$, and this will end the proof. Assuming that $H_{M} \neq E$, we can choose $v_{0} \in E \backslash H_{M}$ and define $H_{0}=H_{M} \bigoplus \mathbb{R} v_{0}, T_{0}: H_{0} \rightarrow F$,

$$
T_{0}\left(h+r v_{0}\right)=T_{H_{M}}(h)+r y_{0}, h \in H_{M}, r \in \mathbb{R}
$$

where $y_{0} \in F$ will be chosen such that $\left(H_{M}, T_{H_{M}}\right) \ll\left(H_{0}, T_{0}\right)$ and $\left(H_{0}, T_{0}\right) \in \mathcal{L}$. This will contradict the maximality of $\left(H_{M}, T_{H_{M}}\right)$ in $\mathcal{L}$. Thus, $H_{M}=E$. To prove that $\left(H_{0}, T_{0}\right) \in \mathcal{L}$ for suitable $y_{0} \in F$, we have to show that

$$
h \in H_{M}, r \in \mathbb{R}, h+r v_{0} \leq x \in E_{+} \Rightarrow T_{H_{M}}(h)+r y_{0} \leq \Phi(x)
$$

For $r=\alpha>0$, multiplying $\alpha^{-1}$ with the relation $h_{1}+\alpha v_{0} \leq x_{1} \in E_{+}$. the above implication becomes

$$
h_{1}+\alpha v_{0} \leq x_{1} \in E_{+} \Rightarrow y_{0} \leq \alpha^{-1}\left(\Phi\left(x_{1}\right)-T_{H_{M}}\left(h_{1}\right)\right), \alpha>0,
$$

and, respectively,

$$
h_{2}+\beta v_{0} \leq x_{2} \in E_{+} \Rightarrow y_{0} \geq \beta^{-1}\left(\Phi\left(x_{2}\right)-T_{H_{M}}\left(h_{2}\right)\right), \beta<0
$$

To have both conditions on $y_{0}$ verified, according to order completeness of $F$, it is necessary and sufficient to prove that

$$
\beta^{-1}\left(\Phi\left(x_{2}\right)-T_{H_{M}}\left(h_{2}\right)\right) \leq \alpha^{-1}\left(\Phi\left(x_{1}\right)-T_{H_{M}}\left(h_{1}\right)\right)
$$

The last inequality may be written as

$$
T_{H_{M}}\left(\alpha^{-1} h_{1}-\beta^{-1} h_{2}\right) \leq \alpha^{-1} \Phi\left(x_{1}\right)-\beta^{-1} \Phi\left(x_{2}\right)
$$

To prove this last inequality, we eliminate $v_{0}$ by adding the previous inequalities, multiplied by $\alpha^{-1}>0$, respectively, by $-\beta^{-1}>0$, as follows

$$
\left(\alpha^{-1} h_{1}+v_{0} \leq \alpha^{-1} x_{1},-\beta^{-1} h_{2}-v_{0} \leq-\beta^{-1} x_{2}\right) \Rightarrow \alpha^{-1} h_{1}-\beta^{-1} h_{2} \leq \alpha^{-1} x_{1}-\beta^{-1} x_{2} \in E_{+},
$$


where $h_{j} \in H_{M}, x_{j} \in E_{+}, j=1,2, \alpha>0, \beta<0$. Since $\left(H_{M}, T_{H_{M}}\right) \in \mathcal{L}$ and $\Phi$ is convex, this further yields

$$
\begin{gathered}
\frac{1}{\alpha^{-1}-\beta^{-1}} T_{H_{M}}\left(\alpha^{-1} h_{1}-\beta^{-1} h_{2}\right)=T_{H_{M}}\left(\frac{\alpha^{-1}}{\alpha^{-1}-\beta^{-1}} h_{1}+\frac{-\beta^{-1}}{\alpha^{-1}-\beta^{-1}} h_{2}\right) \leq \\
\Phi\left(\frac{\alpha^{-1}}{\alpha^{-1}-\beta^{-1}} x_{1}+\frac{-\beta^{-1}}{\alpha^{-1}-\beta^{-1}} x_{2}\right) \leq \\
\frac{\alpha^{-1}}{\alpha^{-1}-\beta^{-1}} \Phi\left(x_{1}\right)+\frac{-\beta^{-1}}{\alpha^{-1}-\beta^{-1}} \Phi\left(x_{2}\right)
\end{gathered}
$$

Thus, the expected inequality follows and the proof is complete.

Here is the variant of the previous Theorem 5, valid for the case when the convex operator $\Phi$ is defined over the entire space $E$.

Theorem 6. Let $E$ be an ordered vector space, $F$ an order complete vector space, $H \subset E$ a vector subspace, $T_{1}: H \rightarrow F$ a linear operator, $\Phi: E \rightarrow F$ a convex operator. The following statements are equivalent:

(a) There exists a positive linear extension $T: E \rightarrow F$ of $T_{1}$ such that $T \leq \Phi$ on $E$;

(b) We have $T_{1}(h) \leq \Phi(x)$ for all $(h, x) \in H \times E$ such that $h \leq x$.

The proof of Theorem 6 is practically the same as that of Theorem 5 .

Remark 2 Given a vector space E, application of Theorem 6 to the very particular case when we endow E with the order relation defined by the equality-relation $\left(E_{+}=\{0\}\right)$, leads to the classical Hahn-Banach extension result (where positivity of $T$ is not involved in any way).

In the end of this subsection, we rewrite Theorem 6 in terms of the corresponding abstract moment problem.

Theorem 7. (Theorem 1 [11]). Let E, F, $\Phi$ be as in Theorem $6,\left\{\varphi_{j}\right\}_{j \in J} \subset E,\left\{y_{j}\right\}_{j \in J} \subset F$ given families. The following statements are equivalent:

(a) There exists a positive linear operator $T: E \rightarrow F$ such that $T\left(\varphi_{j}\right)=y_{j}, \forall j \in J, T \leq \Phi$ on $E$;

(b) For any finite subset $J_{0} \subset J$ and any $\left\{\lambda_{j} ; j \in J_{0}\right\} \subset \mathbb{R}$, the following implication holds true

$$
\sum_{j \in J_{0}} \lambda_{j} \varphi_{j} \leq \psi \in E \Longrightarrow \sum_{j \in J_{0}} \lambda_{j} y_{j} \leq \Phi(\psi)
$$

\subsection{Extending Inequalities via Krein-Milman and Carathéodory's Theorems}

The next results extend an inequality occurring on a small set to a much larger subset.

Theorem 8. Let E be a reflexive Banach lattice, F an order complete Banach lattice in which every topological bounded subset is order-bounded and $y_{n} \uparrow y$ implies $y_{n} \rightarrow y, \Phi: E_{+} \rightarrow F$ a quasiconvex continuous positively homogeneous operator, $T \in B_{+}(E, F)$ a positive linear operator such that $\Phi(e) \leq T(e)$ for all extreme points e of the set $K:=E_{+} \cap B_{1, E}$. Then

$$
\Phi(x) \leq\|x\| \cdot \sup _{e \in E x(K)} \Phi(e) \in F_{+} \forall x \in E_{+}
$$


Proof. Recall that an operator $\Phi$ from a convex subset $C$ of a vector space $E$ to a vector lattice $F$ is called quasiconvex if

$$
\Phi\left((1-t) x_{1}+t x_{2}\right) \leq \sup \left\{\varphi\left(x_{1}\right), \varphi\left(x_{2}\right)\right\}, \forall t \in[0,1], \forall x_{1}, x_{2} \in C
$$

Following the proof (by induction) of Jensen's inequality for real quasiconvex functions, for any convex combination $\sum_{j=1}^{n} \alpha_{j} x_{j}, x_{j} \in C, j=1, \ldots, n$, a quasiconvex operator $\Phi$ verifies

$$
\Phi\left(\sum_{j=1}^{n} \alpha_{j} x_{j}\right) \leq \sup \left\{\Phi\left(x_{1}\right), \ldots, \Phi\left(x_{n}\right)\right\}
$$

See [5] for details, examples and exercises related to this important notion. The set $K:=E_{+} \cap B_{1, E}$ is convex, weakly compact and

$$
K=\operatorname{cl}(\operatorname{co}(\operatorname{Ex}(K)))
$$

holds thanks to Krein-Milman theorem. Let

$$
x_{n}=\sum_{j=1}^{n} \alpha_{j} e_{j} \in \operatorname{co}(\operatorname{Ex}(K)), e_{j} \in \operatorname{Ex}(K), \alpha_{j} \in[0, \infty), j=1, \ldots, n, \sum_{j=1}^{n} \alpha_{j}=1
$$

According to (6) and also using the hypothesis, we infer that

$$
\Phi\left(x_{n}\right) \leq \sup \left\{\Phi\left(e_{1}\right), \ldots, \Phi\left(e_{n}\right)\right\} \leq \sup \left\{T\left(e_{1}\right), \ldots, T\left(e_{n}\right)\right\}
$$

On the other side, any positive linear operator from $E$ to $F$ is continuous, so that the image of the bounded set $K \subset E_{+}$through the positive (bounded) linear operator $T$ is topologically bounded, hence is $o-$ bounded in $F_{+}$. Thus, it results

$$
T(K) \subset\left[0_{F}, y_{0}\right] \text { for some } y_{0} \in F_{+}
$$

From this and also using (7), it results

$$
\Phi\left(x_{n}\right) \leq y_{0}, x_{n} \in \operatorname{co}(E x(K)), n \in \mathbb{N}, n \geq 1
$$

If $x=\lim _{n \rightarrow \infty} x_{n} \in \operatorname{cl}(\operatorname{co}(\operatorname{Ex}(K)))=K$, where $x_{n} \in \operatorname{co}(\operatorname{Ex}(K))$ for all $n \geq 1$, then, thanks to the continuity of $\Phi$, we are leaded to

$$
\begin{gathered}
\Phi(x)=\lim _{n \rightarrow \infty} \Phi\left(x_{n}\right) \leq \lim _{n \rightarrow \infty}\left(\sup \left\{\Phi\left(e_{j}\right) ; j=1, \ldots, n\right\}\right)= \\
\sup _{n \geq 1} \Phi\left(e_{n}\right) \leq \sup _{e \in E x(K)} \Phi(e) \leq \sup _{e \in E x(K)} \mathrm{T}(e) \leq y_{0}, x \in K
\end{gathered}
$$

Since $\Phi$ is positively homogeneous, application of this evaluation to $x /\|x\| \in K$, for all $x \in E_{+}, x \neq 0_{E}$, yields

$$
\Phi(x) \leq\|x\| \cdot \sup _{e \in E x(K)} \Phi(e) \leq\|x\| y_{0}, x \in E_{+}
$$

This concludes the proof.

Theorem 9. Let $E$ be an order complete normed vector lattice, $K \subset E$ a finite dimensional compact subset, $\left(\Phi_{n}\right)_{n \geq 0}$ a sequence of continuous sublinear operators from $E$ to $E$, such that for each $x \in E$, there exists $\widetilde{\Phi}(x):=\lim _{n \rightarrow \infty} \Phi_{n}(x) \in E$. Assume that for each $n \in \mathbb{N}$, there exists an affine operator $T_{n}$ from $E$ to $E$, such that $T_{n}(x) \leq \Phi_{n}(x) \forall x \in E$ and there exists

$$
\widetilde{T}(e):=\lim _{n \rightarrow \infty} T_{n}(e)=e \forall e \in E x(K)
$$


Then $x \leq \widetilde{\Phi}(x) \forall x \in \operatorname{Cone}(K)$, where Cone $(K)$ is the convex cone generated by $(c o(K)) \cup\left\{0_{E}\right\}$.

Proof. It is known that for any finite dimensional compact $K$, its convex hull $c o(K)$ is compact too (the proof of this assertion is based on Carathéodory's theorem, which leads to a way of expressing $c o(K)$ as image of a compact (finite dimensional) subset through a continuous mapping). Let $p$ be the dimension of the linear variety generated by $K$ (and by $c o(K)$ ) and $x \in c o(K)$. Assume that $p \geq 2$. Due to Carathéodory's theorem, there exist at most $p+1$ extreme points in the compact (convex) subset $\operatorname{co}(K)$, say $e_{1}, \ldots, e_{p+1}$ and $\left\{\alpha_{1}, \ldots, \alpha_{p+1}\right\} \subset[0, \infty), \sum_{j=1}^{p+1} \alpha_{j}=1$, such that $x=\sum_{j=1}^{p+1} \alpha_{j} e_{j}$. In addition, it is known that any extreme point of $c o(K)$ is (an extreme) point of $K$. From the hypothesis we infer that

$$
T_{n}(x)=\sum_{j=1}^{p+1} \alpha_{j} T_{n}\left(e_{j}\right) \rightarrow \sum_{j=1}^{p+1} \alpha_{j} \widetilde{T}\left(e_{j}\right)=\sum_{j=1}^{p+1} \alpha_{j} e_{j}=x, n \rightarrow \infty
$$

Thus, there exists $\widetilde{T}(x)=x \forall x \in c o(K)$. On the other side, the positive cone $E_{+}$of the space $E$ is closed and we have assumed that $\Phi_{n}(x)-T_{n}(x) \in E_{+}$for all $x \in E$ and all $n \in \mathbb{N}$. Passing to the limit, one obtains

$$
\widetilde{\Phi}(x)-\widetilde{T}(x)=\widetilde{\Phi}(x)-x \in E_{+} \forall x \in \operatorname{co}(K) \Leftrightarrow \widetilde{\Phi}(x) \geq x \forall x \in c o(K)
$$

(Since $\widetilde{T}$ is the pointwise limit of affine operators, it is affine on $c o(K)$; a Hahn-Banach argument shows that it has an affine extension from the whole space $E$ to $E$. We denote this extension by $\widetilde{T}$ too). Recall that if $0_{E}$ is not an element of $c o(K)$, then

$$
C:=\operatorname{Cone}(K)=\{\alpha x ; \alpha \in[0, \infty), x \in \operatorname{co}(K)\}
$$

It is easy to see that in this case: $C \cap(-C)=\left\{0_{E}\right\}$. Now (8) and (9) yield

$$
\widetilde{\Phi}(\alpha x)=\alpha \widetilde{\Phi}(x) \geq \alpha x \forall \alpha \in[0, \infty), \forall x \in c o(K) \Leftrightarrow \widetilde{\Phi}(w) \geq w \forall w \in C
$$

If $0_{E} \in \partial(c o(K)) \backslash r i(c o(K))$, then $C$ could satisfy the condition $C \cap(-C)=\left\{0_{E}\right\}$, or $C \cap(-C)$ might be a nonzero vector subspace (here $r i(c o(K))$ is the relative interior of $c o(K))$. When $0_{E} \in r i(c o(K)), C$ is a $p$-dimensional vector subspace of $E$. In both these last two cases, the conclusion of the theorem still holds true, following the same proof as in the first case, (when $0_{E}$ was not an element of $c o(K)$ ). This concludes the proof.

Remark 3. Assume now that $0_{E}$ is not an element of $c o(K)$. Then there exists a strictly positive linear continuous form $T$ on $E$ endowed with the order relation defined by $C$, such that $\|T\|=1$, and a constant $\beta>0$ with

$$
\inf _{x \in \operatorname{co}(K)} T(x)=\beta=T(e) \text { for some } e \in \operatorname{Ex}(\operatorname{co}(K))
$$

Indeed, denote by $d_{0}:=d\left(0_{E}, c o(K)\right)>0, V:=B_{d_{0}}\left(0_{E}\right)=\left\{x \in E ;|x|<d_{0}\right\}$. Then $V$ is a convex open neighborhood of the origin, which does not intersect $c o(K)$. By geometric form of Hahn-Banach theorem, there exists a closed hyperplane separating $V$ from $c o(K)$, and not intersecting $V$ i.e., there exists a linear continuous form $T$ on $E$ such that $0<\sup T(V) \leq \beta \leq \inf T(\operatorname{co}(K))$. In particular, $T(x) \geq \beta>0 \forall x \in c o(K) \Rightarrow T(w)>0 \forall w \in C \backslash\left\{0_{E}\right\}$. When $T$ is strictly positive and for any $\gamma>0$, the set $B=\{x \in C ; T(x)=\gamma\}$ is a compact base for $C$. Now scaling $T$ by a positive scalar we obtain a new strictly continuous positive form (which we also denote by $T$ ) with the special property

$$
0<\sup T(V)=d_{0}=\inf T(\operatorname{co}(K)), T(x)<d_{0} \forall x \in V
$$


It results

$$
T\left(d_{0} \frac{x}{\|x\|+\varepsilon}\right)<d_{0} \forall \varepsilon>0, \forall x \in X \Rightarrow|T(x)| \leq\|x\|, \forall x \in E \Rightarrow\|T\| \leq 1
$$

Let $x_{\star} \in \operatorname{co}(K) \cap \partial V$ be such that $T\left(x_{\star}\right)=d_{0}$. Then $\left\|x_{\star}\right\|=d_{0}$ and $T\left(\frac{x_{\star}}{\left\|x_{\star}\right\|}\right)=1$. Thus, $\|T\|=1$. Consider the hyperplane $H:=\left\{x ; T(x)=d_{0}\right\}$ and the base $B=H \cap C$. Then the distance

$$
d\left(0_{E}, H\right)=\frac{\left|T(0)-d_{0}\right|}{\|T\|}=d_{0}=d\left(0_{E}, c o(K)\right)
$$

as expected ( $H$ and $B$ are separating $c l(V)$ and $c o(K)$, but they are "tangent" to both these closed convex subsets). If $E$ is a real Hilbert space and $T$ has the properties from above, then $x_{\star}$ is the orthogonal (or metric) projection of $0_{E}$ to $c o(K)$. Consequently,

$$
\left\|x_{\star}\right\|=d\left(0_{E}, \operatorname{co}(K)\right)=d_{0}, x_{\star} \perp H
$$

Having in mind the idea of the proof of Riesz representation theorem for linear continuous forms on a Hilbert space, it results that $T$ is represented by a vector which is collinear to $x_{\star}$. Since $\|T\|=1$, we have to normalize $x_{\star}$. It results

$$
T(x)=<\frac{x_{\star}}{\left\|x_{\star}\right\|}, x>=<\frac{x_{\star}}{d_{0}}, x>\forall x \in E
$$

\section{Discussion}

The above results point out four directions for applications of generalizations of Hahn-Banach theorem, mentioned in the end of the Introduction. On the other hand, as we have already seen in the Introduction, solving multidimensional moment problems (and other problems involving inequalities or positivity of linear operators) in terms of signatures of quadratic forms is a difficult task, simply since nonnegative polynomials on closed subsets of $\mathbb{R}^{n}, n \geq 2$ generally are not expressible by means of sums of squares. When working on Cartesian products of closed (in general unbounded) intervals, this difficulty can be partially or completely solved by means of polynomial approximation on such subsets. Namely, one approximates any nonnegative continuous compactly supported function by a sequence of special nonnegative polynomials $p_{m}, m \in \mathbb{N}, p_{m}\left(t_{1}, \ldots, t_{n}\right)=p_{m, 1}\left(t_{1}\right) \cdots p_{m, n}\left(t_{n}\right)$, where $p_{m_{j}}$ is positive on a whole closed interval $I_{j}, j=1, \ldots, n$. Since any nonnegative polynomial of one real variable on a closed interval is expressible in terms of sums of squares, passing to the limit by means of Fatou lemma, one goes from inequalities on such expressible polynomials to inequalities on arbitrary nonnegative continuous compactly supported functions. This could be the aim of a future review paper.

Going back to the results of the present work, notice that an important particular case of a continuous convex operator is that of a sublinear continuous operator, which will denoted by $P$. In this respect, we mention the following two remarks.

Remark 4. Let E be a Banach space, F an order complete Banach space, $P: E \rightarrow F$ a continuous sublinear operator. Then for each $x_{0} \in E$ and any $T \in \partial_{x_{0}} P$, we have $T\left(x_{0}\right)=P\left(x_{0}\right), T \in \partial P$. Consequently, $\partial P=\partial_{0} P=\bigcup_{x_{0} \in E} \partial_{x_{0}} P$.

Indeed, if $T \in \partial_{x_{0}} P$, then, by definition, $P(x)-P\left(x_{0}\right) \geq T(x)-T\left(x_{0}\right)$ for all $x \in E$. Writing this for $r x$ instead of $x, r \in[0, \infty)$, one obtains (via positively homogeneity)

$$
r(P(x)-T(x)) \geq P\left(x_{0}\right)-T\left(x_{0}\right), x \in E
$$


If $r=0$ we infer that $T\left(x_{0}\right) \geq P\left(x_{0}\right)$. On the other hand, dividing by $r>0$ and passing to the limit as $r \rightarrow \infty$ we obtain $P(x) \geq T(x), x \in E$. In particular, we have obtained $T\left(x_{0}\right)=P\left(x_{0}\right)$ and $T(x) \leq P(x), x \in E$. The conclusion follows. For notations and detailed information see [14] or any other reference concerning subdifferentials of convex operators.

Remark 5. Let $E, F$ be Banach lattices and, $P: E \rightarrow F$ a continuous sublinear operator. If $T: E \rightarrow F$ is a linear operator such that $T \leq P$ on $E$, then $\|T\| \leq\|P\|$ in the following two cases at least: (1) $P$ is isotone; (2) $P$ is symmetric $(P(x)=P(-x) \forall x \in E)$.

Indeed, in case (1), if $P$ is isotone on $E$, then

$$
T(x) \leq P(x) \leq P(|x|),-T(x)=T(-x) \leq P(-x) \leq P(|x|)
$$

implies $|T(x)| \leq P(|x|), x \in E$, that further yields

$$
\|T(x)\| \leq\|P(|x|)\| \leq\|P\|\|\mid\| x\|, x \in E \Longrightarrow\| T\|\leq\| P \|
$$

In case (2), assuming that $P$ is symmetric, we obtain

$$
\begin{gathered}
T(x) \leq P(x),-T(x)=T(-x) \leq P(-x)=P(x) \Longrightarrow \\
|T(x)| \leq P(x) \Longrightarrow\|T(x)\| \leq\|P(x)\|, x \in E \Longrightarrow\|T\| \leq\|P\|
\end{gathered}
$$

The preceding remark is still valid for convex symmetric operators $P: E \rightarrow F$ verifying

$$
\sup _{\|x\| \leq 1}\|P(x)\| \leq M<\infty, P(\mathbf{0})=\mathbf{0}
$$

In this case, if $T$ is linear, dominated by $P$ on the entire domain space $E$, then $\|T\| \leq M$. Indeed, similar to the case (2) from above, we have: $\|T(x)\| \leq\|P(x)\|, x \in E$. This leads to

$$
\|T\|=\sup _{\|x\| \leq 1}\|T(x)\| \leq \sup _{\|x\| \leq 1}\|P(x)\| \leq M
$$

Examples of such convex operators which are not sublinear can be constructed defining

$$
P(x)=\|x\|^{p} y_{1}, x \in E, p \in(1, \infty),
$$

where $y_{1} \in F_{+}, y_{1} \neq 0$. For the above operator, the constant $M$ equals $\left\|y_{1}\right\|$.

Funding: This research received no external funding.

Conflicts of Interest: The author declares no conflict of interest.

\section{References}

1. Akhiezer, N.I. The Classical Moment Problem and Some Related Questions in Analysis; Oliver \& Boyd: Edinburgh, UK; London, UK, 1965.

2. Krein, M.G.; Nudelman, A.A. Markov Moment Problem and Extremal Problems; American Mathematical Society: Providence, RI, USA, 1977.

3. Schmüdgen, K. Graduate Texts in Mathematics. In The Moment Problem; Springer: Berlin/Heidelberg, Germany; New York, NY, USA, 2017.

4. Cristescu, R. Ordered Vector Spaces and Linear Operators; Academiei: Bucharest, Romania; Abacus Press: Kent, UK, 1976.

5. Niculescu, C.P.; Persson, L.-E. Convex Functions and Their Applications. A Contemporary Approach, 2nd ed.; (CMS Books in Mathematics); Springer: New York, NY, USA, 2018; Volume 23. 
6. Phelps, R.R. Lectures on Choquet's Theorem, 2nd ed.; Springer: Berlin/Heidelberg, Germany, 2001.

7. Schaefer, H.H. Topological Vector Spaces. Third Printing Corrected; Springer: Berlin/Heidelberg, Germany; New York, NY, USA, 1971.

8. Olteanu, O. Recent Results on Markov Moment Problem, Polynomial Approximation and Related Fields in Analysis; Generis Publishing: Chişinău, Moldova, 2020.

9. Olteanu, O. Convexité et prolongement d'opérateurs linéaires. C. R. Acad. Sci. Paris 1978, 286, 511-514.

10. Olteanu, O. Théorèmes de prolongement d'opérateurs linéaires. Rev. Roum. Math. Pures Appl. 1983, 28, 953-983.

11. Olteanu, $\mathrm{O}$. Application de théorèmes de prolongement d'opérateurs linéaires au problème des moments e à une généralization d'un théorème de Mazur-Orlicz. C. R. Acad. Sci. Paris 1991, 313, 739-742.

12. Olteanu, O. Applications of a general sandwich theorem for operators to the moment problem. Rev. Roum. Math. Pures Appl. 1996, 41, 513-521.

13. Ambrozie, C.; Olteanu, O. A sandwich theorem, the moment problem, finite-simplicial sets and some inequalities. Rev. Roum. Math. Pures Appl. 2004, 49, 189-210.

14. Niculescu, C.P.; Olteanu, O. A note on the isotonic vector-valued convex functions. arXiv 2005, arXiv:2005.01088.

15. Niculescu, C.P.; Olteanu, O. From the Hahn-Banach extension theorem to the isotonicity of convex functions and the majorization theory. RACSAM 2020, 114, 1-19. [CrossRef]

16. Valadier, M. Sous-Différentiablité de fonctions convexes à valeurs dans un espace vectoriel ordonné. Math. Scand. 1972, 30, 65-74. [CrossRef]

17. Zowe, J. Linear Maps Majorized by a Sublinear Map. Arch. Math. 1975, 26, 637-645. [CrossRef]

18. Zowe, J. Sandwich Theorems for Convex Operators with Values in an Ordered Vector Space. J. Math. Anal. Appl. 1978, 66, 282-296. [CrossRef]

19. Kutateladze, S.S. Convex operators. Russ. Math. Surv. 1979, 34, 181-214. [CrossRef]

20. Haviland, E.K. On the momentum problem for distributions in more than one dimension. Amer. J. Math. 1936, 58, 164-168. [CrossRef]

21. Marshall, M. Polynomials non-negative on a strip. Proc. Am. Math. Soc. 2010, 138, 1559-1567. [CrossRef]

22. Choquet, G. Le problème des moments. In Séminaire d'Initiation à l'Analise; Institut H. Poincaré: Paris, France, 1962.

23. Berg, C.; Christensen, J.P.R.; Jensen, C.U. A remark on the multidimensional moment problem. Math. Ann. 1979, 243, 163-169. [CrossRef]

24. Berg, C.; Durán, A.J. The fixed point for a transformation of Hausdorff moment sequences and iteration of a rational function. Math. Scand. 2008, 103, 11-39. [CrossRef]

25. Berg, C.; Beygmohammadi, M. On fixed point in the metric space of normalized Hausdorff moment sequences. Rend. Circ. Mat. Palermo. 2010, 82, 251-257.

26. Cassier, G. Problèmes des moments sur un compact de $\mathbb{R}^{\mathrm{n}}$ et décomposition des polynômes à plusieurs variables. J. Funct. Anal. 1984, 58, 254-266. [CrossRef]

27. Fuglede, B. The multidimensional moment problem. Expo. Math. 1983, 1, 47-65.

28. Schmüdgen, K. The moment problem for compact semi-algebraic sets. Math. Ann. 1991, 289, $203-206$. [CrossRef]

29. Gosse, L.; Runborg, O. Existence, uniqueness, and a constructive solution algorithm for a class of finite Markov moment problems. SIAM J. Appl. Math. 2008, 68, 1618-1640. [CrossRef]

30. Kleiber, C.; Stoyanov, J. Multivariate distributions and the moment problem. J. Multivar. Anal. 2013, 113, 7-18. [CrossRef]

31. Stoyanov, J.; Lin, G.D. Hardy's condition in the moment problem for probability distributions. Theory Probab. Appl. 2013, 57, 699-708. [CrossRef]

32. Lemnete, L. An operator-valued moment problem. Proc. Amer. Math. Soc. 1991, 112, 1023-1028. [CrossRef]

33. Lemnete-Ninulescu, L.; Zlătescu, A. Some new aspects of the L-moment problem. Rev. Roum. Math. Pures Appl. 2010, 55, 197-204.

34. Putinar, M. Positive polynomials on compact semi-algebraic sets. Indiana Univ. Math. J. 1993, 42, 969-984. [CrossRef]

35. Vasilescu, F.H. Spectral measures and moment problems. In Spectral Analysis and Its Applications; (Ion Colojoară Anniversary Volume); Theta: Bucharest, Romania, 2003; pp. 173-215. 
36. Mihăilă, J.M.; Olteanu, O.; Udrişte, C. Markov-type and operator-valued multidimensional moment problems, with some applications. Rev. Roum. Math. Pures Appl. 2007, 52, 405-428.

37. Mihăilă, J.M.; Olteanu, O.; Udrişte, C. Markov-type moment problems for arbitrary compact and some noncompact Borel subsets of $\mathbb{R}^{\mathrm{n}}$. Rev. Roum. Math. Pures Appl. 2007, 52, 655-664.

38. Norris, D.T. Optimal Solutions to the $\mathrm{L}_{\infty}$ Moment Problem with Lattice Bounds. Ph.D. Thesis, University of Colorado, Denver, CO, USA, 2003.

39. Olteanu, O. New results on Markov moment problem. Int. J. Anal. 2013. [CrossRef]

40. Olteanu, O. Moment problems on bounded and unbounded domains. Int. J. Anal. 2013. [CrossRef]

41. Olteanu, O. Applications of Hahn-Banach principle to the moment problem. Poincare J. Anal. Appl. 2015, 1, 1-28. [CrossRef]

42. Olteanu, O. Mazur-Orlicz theorem in concrete spaces and inverse problems related to the moment problem. UPB Sci. Bull. Ser. A 2017, 79, 151-162.

43. Olteanu, O.; Mihăilă, J.M. On Markov moment problem and Mazur-Orlicz theorem. Open Access Libr. J. 2017, 4, 1-10. [CrossRef]

44. Olteanu, O.; Mihăilă, J.M. Extension and decomposition of linear operators dominated by continuous increasing sublinear operators. UPB Sci. Bull. Ser. A 2018, 80, 133-144.

45. Olteanu, O.; Mihăilă, J.M. Markov moment problem in concrete spaces revisited. MathLAB J. 2020, 5, 82-91.

46. Olteanu, O. New results on extension of linear operators and Markov moment problem. MathLAB J. 2020, 5, 143-154.

47. López Montiel, P.; Galán Ruiz, M. Revisiting the Hahn-Banach theorem and nonlinear infinite programming. J. Math. Anal. Appl. 2017, 455, 1037-1050.

48. Galán Ruiz, M. A version of the Lax-Milgram theorem for locally convex spaces. J. Convex Anal. 2009, 16, 993-1002.

49. Simons, S. From Hahn-Banach to monotonicity, 2nd ed.; Lecture Notes in Mathematics 1693; Springer: New York, NY, USA, 2008.

50. Simons, S. The asymmetric sandwich theorem. J. Convex Anal. 2013, 20, 107-124.

51. Simons, S. Bootstrapping the Mazur-Orlicz-König theorem and the Hahn-Banach-Lagrange theorem. J. Convex Anal. 2018, 25, 691-699. 